\title{
RECAUDACIÓN DE PATENTES Y SU INFLUENCIA EN EL PRESUPUESTO DE ENTIDADES GUBERNAMENTALES: UN ANÁLISIS DE REVISIÓN POR SECTORES
}

\author{
Salazar Nicole', Vega Andrea² y Parreño Carlos ${ }^{3}$. \\ \{nasalazar4 $4^{1}$, javega14 ${ }^{2}$, caparrenio ${ }^{3}$ \}@espe.edu.ec \\ https://orcid.org/0000-0001-9116-29421', https://orcid.org/0000-0002-6946-2487², \\ https://orcid.org/0000-0002-8908-5607³. \\ Universidad de las Fuerzas Armadas, ESPE
}

Sangolquí - Ecuador

Recibido (02/10/20), Aceptado (20/11/20)

\begin{abstract}
Resumen: Al analizar los aportes sectoriales sobre la recaudación de impuestos, en este caso el análisis de la patente municipal evidenció que las organizaciones poseen escasos conocimientos sobre este tributo. Por tal razón, el objetivo del estudio es determinar su incidencia en el presupuesto de los Gobiernos Autónomos Descentralizados. La investigación posee un orden cuantitativo de alcance correlacional, pues se determinó la relación de la recaudación presupuestada frente la recaudada e ingresos totales en el año 2017. Mediante el coeficiente de correlación de Pearson se midió la relación de las variables y se identificó una relación lineal directa. Se concluye que, si existe incidencia entre la recaudación del impuesto de patentes y el presupuesto de los municipios. De igual forma, los sectores que han contribuido en la recaudación han sido el sector financiero y comercial.
\end{abstract}

Palabras Clave: Patente municipal, ingresos tributarios, Presupuesto.

\section{PATENT COLLECTION AND ITS INFLUENCE ON THE BUDGET OF GOVERNMENT ENTITIES. A REVIEW ANALYSIS BY SECTORS}

\begin{abstract}
When analyzing the sector contributions on the collection of taxes, in this case the analysis of the municipal license showed that the organizations have little knowledge about this tax. For this reason, the objective of the study is to determine its impact on the budget of the Decentralized Autonomous Governments. The research has a quantitative order of correlational scope, since the relationship of the budgeted collection versus the collected and total income in 2017 was determined. Using the Pearson correlation coefficient, the relationship of the variables was measured and a linear relationship was identified direct. It is concluded that, if there is an incidence between the collection of the patent tax and the budget of the municipalities. Similarly, the sectors that have contributed to the collection have been the financial and commercial sector.
\end{abstract}

Keywords: Municipal patent, tax revenue, Budget. 


\section{I.INTRODUCCIÓN}

En la actualidad, las patentes se han convertido en un derecho para realizar actividades económicas [1]. Esto genera una obligación tributaria controlada por parte de las entidades gubernamentales, específicamente los Gobiernos Autónomos Descentralizados [2]; lo que da relevancia al estudio de la recaudación de patentes y su influencia en el presupuesto de entidades gubernamentales, por medio de un análisis de revisión por sectores [3]- [4].

La recaudación eficaz del impuesto a las patentes es trascendental porque permite la obtención de mayores ingresos locales para la ejecución presupuestaria y así satisfacer en gran manera las necesidades de la sociedad [5].

El bajo nivel de recaudación del impuesto se debe a múltiples razones, entre ellas: la ineficiente cultura tributaria que parte del desconocimiento en la tramitología y legislación del impuesto que provoca la evasión tributaria por ignorancia o intención directa, las escasas fuentes de trabajo ocasionan que los contribuyentes obtengan bajos ingresos y se incremente la informalidad, en consecuencia, un gran número de patentes municipales no registradas. En la entidad gubernamental, si no existe el cotejo de la base de datos del SRI y el Municipio se tendrá como resultado un catastro desactualizado [6]. Por eso, la escasa recaudación del impuesto a las patentes afecta de manera directa a la disminución en el presupuesto de los municipios [7].

Se pretende plantear soluciones con el propósito de lograr mayores recaudaciones y un presupuesto fortalecido por una alta participación de este impuesto dentro de los Gobiernos Autónomos. El objetivo general del estudio es analizar los aportes sectoriales sobre la recaudación de patentes mediante una investigación bibliográfica para determinar su incidencia en el presupuesto de entidades gubernamentales. Se emplea una investigación de enfoque cuantitativo no experimental, alcance correlacional, modalidad bibliográfica-documental de corte transversal para relacionar las variables, aplicando el coeficiente de correlación de Pearson y el análisis de regresión lineal mediante el software estadístico R-Studio.

La estructura manifestada se lleva a cabo desde un breve resumen y las palabras clave, la introducción del tema, el desarrollo que detallará el comportamiento de las variables, la metodología aplicada para la obtención de los resultados, la exposición de las conclusiones y finalmente las fuentes bibliográficas de donde parte la investigación.

\section{II.DESARROLLO}

De acuerdo al contexto de los tributos, formaron parte de las primeras leyes que se aplicaron en Egipto, China, España y Mesopotamia [4]. Uno de los más importantes en el tiempo es el diezmo, tributo que se debía pagar a la iglesia. De similar forma, los impuestos en algunas naciones y ciudades antiguas eran considerados como una forma de dominación sobre un pueblo.

En el Ecuador, el cobro de los estancos se establece sobre los artículos de primera necesidad como la sal, tabaco, aguardiente y pólvora entre los años 1830 y 1930 , cobros que formaron parte de una fuente de enriquecimiento de particulares, aunque siempre hubo el rechazo del pueblo a la tributación [8]. Debido a la intervención del Fondo Monetario Internacional en 1980 se adopta el modelo empresarial de desarrollo consolidado al aplicar reformas tributarias. En 1983 se pudo obtener un incremento del $5 \%$ y $6 \%$ en el impuesto de transacciones mercantiles. Al pasar los años, este efecto siguió aumentando desde un $6 \%$ hasta un $10 \%$.

Al finalizar el año 1989, ya existía un sistema consolidado para el cobro de impuestos como el Impuesto de Consumos Especiales (ICE), la tasa del impuesto a la renta (IR) personal y demás [9]. Si bien es cierto, existen ventajas y desventajas a través del tiempo durante proceso de acogida tributaria dentro de las sociedades. Razón por la cual, los países han fortalecido sus sistemas de control y estrategias para la correcta distribución de sus ingresos tributarios como no tributarios mediante la administración pública [10].

Las entidades gubernamentales son aquellas que conforman el sector público dentro de un estado, es decir, están bajo el control del gobierno. El Sistema Nacional de Finanzas Públicas clasifica a las entidades gubernamentales como parte del sector público financiero y sector público no financiero, para lo cual, los Gobiernos Autónomos Descentralizados encargados del cobro del impuesto a las patentes, forman parte del Gobierno General, que por la estructura pertenece al Sector Público no financiero [3] (Ver figura 1). 


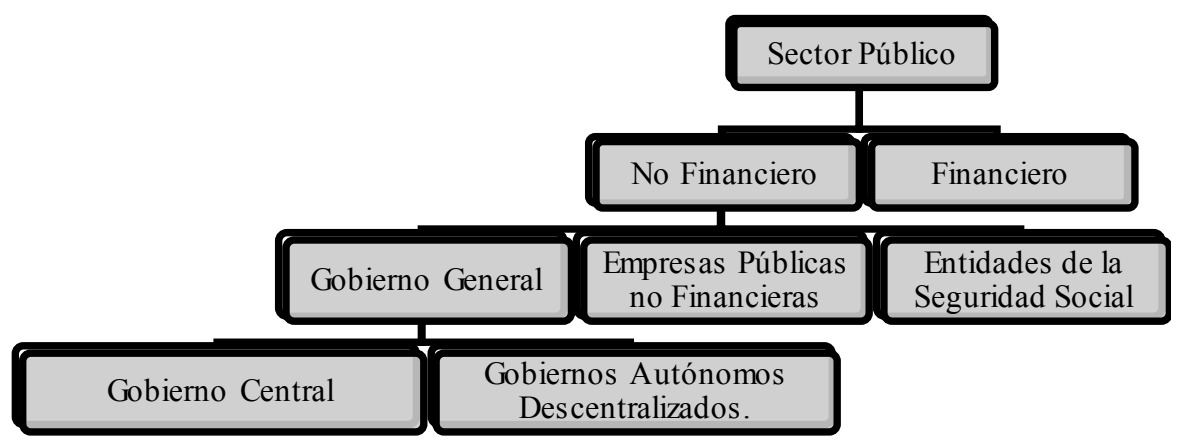

Fig. 1. Diagrama de la conformación del Sector Público del Estado Ecuatoriano

\section{A.Tributo}

Los tributos son un instrumento de la política fiscal de un país. Esta es la principal fuente de ingreso del Estado y demás entidades acreedoras, permitiendo afrontar el gasto público [11]. Los tributos según el Código Orgánico Tributario se clasifican en impuestos, tasas y contribuciones especiales o de mejora. Los impuestos financian servicios públicos; las tasas financian servicios públicos que benefician a determinados contribuyentes y las contribuciones son aquellos tributos que financian gastos en general [12]. Para los Gobiernos Autónomos Descentralizados los tributos se han convertido en la aportación principal a su presupuesto, uno de ellos son las patentes municipales [13].

\section{B.Impuesto de la patente}

En el Ecuador, el impuesto a la patente municipal comienza en el año 1997 publicado en el Registro Oficial Suplemento No. 331 en la Ley Orgánica de Régimen Municipal, en la cual menciona, disposiciones legales para ejecutar la aplicación y cobro del impuesto [5]. El impuesto de patentes, siendo un tributo de carácter obligatorio para personas naturales, jurídicas y sociedades que realizan actividades económicas de orden comercial, industrial, financiera, inmobiliaria y profesionales. Es establecido como un instrumento de política tributaria que se aplica bajo el Código Orgánico de Organización Territorial, Autonomía y Descentralización (COOTAD) [7].

El impuesto a la patente, al ser uno de los principales tributos para los Gobiernos Autónomos Descentralizados forman parte del ingreso corriente, sobre todo aquellas que pertenecen a los tres primeros sectores o actividades; comercial, industrial y financiera [13]. Los contribuyentes primero deben estar registrados en la municipalidad para proceder a la obtención de la patente anual siguiendo el proceso de recaudación normal [2].

Los Consejos Cantonales tienen el derecho de re- caudar y administrar el impuesto mediante ordenanzas para financiar las obras, proyectos y solventar el gasto público dentro de una circunscripción territorial [7]. El monto mínimo es de diez dólares y el máximo de veinte y cinco mil dólares americanos, dependiendo del patrimonio de los contribuyentes, si existiera casos de pérdidas en el Servicio de Rentas Internas habrá la posibilidad de la reducción según el artículo 549 del Código Orgánico de Organización Territorial, Autonomía y Descentralización (COOTAD) [2].

Al momento de la recaudación se ha determinado que existen varias dificultades, tanto de parte interna como externa de la entidad gubernamental; ya que, los contribuyentes no tienen la cultura tributaria adecuada, lo que afecta al presupuesto establecido por cada uno de los municipios. Un control adecuado en el proceso permitirá cumplir las metas establecidas, lo que provocará la disminución del riesgo en la ejecución del presupuesto y en el manejo de recursos [13].

La obligación de contraer una patente y pagar su impuesto no incluye a las actividades que pertenezcan al sector agrícola, pecuario, acuícola, o plantaciones forestales los cuales no son sujetos de cobro por parte de ningún Gobierno Autónomo Descentralizado. Mientras que, las personas exentas del impuesto a las patentes son únicamente los Artesanos Calificados [2].

\section{C.El Presupuesto de los Gobiernos Autónomos Des- centralizados}

Los Gobiernos Autónomos Descentralizados gozan de autonomía política, administrativa y financiera [10]. El plan del presupuesto es aprobado por el Órgano Legislativo y de Fiscalización, para ello, debe mostrar sus objetivos, metas y plazos en un periodo desde el 01 de enero hasta el 31 de diciembre. Por tal razón, tendrán que formular el fondo general de sus ingresos para atender sus gastos; la estructura y el proceso debe ser ajustada a los lineamientos Código Orgánico de Organización Territorial, Autonomía y Descentralización, a la regla- 
mentación del Gobierno Central y la propia normativa del municipio (Ver figura 2) [2].

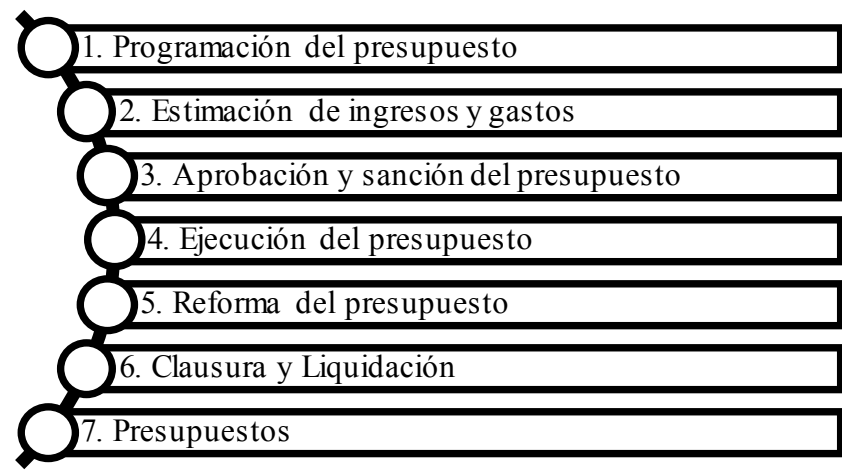

Fig. 2. Proceso Presupuestario.

\section{D.Impuesto de Patentes y Presupuesto de Gobiernos Autónomos Descentralizados}

La patente como obligación tributaria fiscal sirve para el financiamiento del presupuesto de los diferentes Gobiernos Autónomos Descentralizados [6], por tanto, el control eficiente del proceso de recaudación, permite cumplir las metas establecidas para que no exista afectación en el presupuesto asignado y que la ejecución de las actividades satisfagan las necesidades de la sociedad [13].

Al tomar en cuenta. los títulos por los que se dividen los ingresos, la recaudación de las patentes es de naturaleza tributaria, y pertenece al capítulo 1 que trata sobre los impuestos. En cuanto al gasto, se debe determinar si es corriente o de inversión, mostrándose desglosado uniformemente en las partidas. Además, se conoce que existen gastos para servicios: generales, sociales, comunales, económicos e inclasificables, para lo cual, el municipio es capaz de prescindir de cualquier programa de acuerdo a sus necesidades [2].

\section{E.Sectores Económicos del Ecuador}

El Directorio de Empresas y Establecimientos (DIEE) permite conocer la información sincronizada entre el Servicio de Rentas Internas y el Instituto Ecuatoriano de Seguridad Social (IESS); a través de la sistematización de los registros administrativos de empresas públicas y privadas, existe la actualización constante de la información estadística empresarial debido a un conjunto de datos relacionados que facilitan sus actividades, necesidades fiscales, tributarias, entre otros. También, permite conocer el universo de empresas y establecimientos registrados en un periodo determinado, su cobertura cuantitativa, geográfica y de actividad económica; por tanto, cuenta con otras fuentes de información como: Ministerio de Educación, Ministerio de Turismo, Superintendencia de Bancos y Encuestas Internas del INEC [14].

Las empresas se pueden clasificar:

1)El tipo de unidad legal o forma constitucional: personas naturales o jurídicas.

2)El tamaño empresarial determinado por: volumen de ventas anuales y número de personas afiliadas.

3)La actividad económica: el Directorio de Empresas y Establecimientos (DIEE) simplifica la estructura sectorial de las actividades de la Clasificación Industrial Internacional Uniforme (CIIU) en los siguientes sectores económicos [14] (Ver figura 3).

1. Agricultura, ganadería, silvicultura y pesca.
2. Explotación de minas y canteras.
3. Industrias manufactureras.
5. Comercio.
6. Servicios

\section{Fig. 3. Sectores Económicos.}

\section{III.METODOLOGÍA}

El análisis de los aportes sectoriales por recaudación del impuesto de patentes municipales es una investigación de enfoque cuantitativo no experimental, debido a que las variables no se manipularon, sino que, adopta el alcance correlacional que establece la relación positiva o negativa entre sí. Para conocer su comportamiento, se utilizó la modalidad bibliográfica-documental de corte transversal para obtener la información necesaria que contribuya al conocimiento de la influencia de la recaudación de patentes como tributo en los Gobiernos Autónomos Descentralizados y relacionarlo con el análisis de revisión por sectores en el Ecuador dentro de un periodo determinado.

De tal manera, para la obtención de datos se utilizó las cédulas presupuestarias de ingresos del año 2017 del impuesto de patentes, en una población de 221 Gobiernos Autónomos Descentralizados de Ecuador con una muestra de 45 entidades gubernamentales que contaron con la información necesaria en cada página web oficial sección transparencia (Ver tabla I). 
TABLA I. Muestra de Gobiernos Autónomos Descentralizados del Ecuador 2017

\begin{tabular}{|c|c|c|c|c|c|c|}
\hline \multirow{2}{*}{$\begin{array}{l}\text { Entidad Gubernamental } \\
\text { G.A.D. Municipal dePucará }\end{array}$} & \multicolumn{2}{|c|}{ Presupuestado } & \multicolumn{2}{|c|}{ Ingresos Totales } & \multicolumn{2}{|c|}{ Recaudado } \\
\hline & $\$$ & $38.400,00$ & $\$$ & $3.144 .443,37$ & $\$$ & $49.061,74$ \\
\hline G.A.D. Municipal de Guachapalá & $\$$ & $4.397,92$ & $\$$ & $2.132 .063,91$ & $\$$ & $5.937,18$ \\
\hline G.A.D. Municipal de Chordeleg & $\$$ & $33.140,59$ & $\$$ & $4.924 .455,05$ & $\$$ & $34.669,44$ \\
\hline G.A.D. Municipal de Sígsig & $\$$ & $120.000,00$ & $\$$ & $9.535 .094,70$ & $\$$ & $84.831,01$ \\
\hline G.A.D. Municipal de Echeandia & $\$$ & $40.761,17$ & $\$$ & $2.944 .100,36$ & $\$$ & $37.858,34$ \\
\hline G.A.D. Municipal de Bolivar & $\$$ & $37.540,05$ & $\$$ & $6.549 .396,26$ & $\$$ & $52.719,58$ \\
\hline G.A.D. Municipal de Montufar & $\$$ & $202.016,66$ & $\$$ & $354.092,80$ & $\$$ & $2.492,71$ \\
\hline G.A.D. Municipal dePenipe & $\$$ & $5.000,00$ & $\$$ & $9.365 .029,18$ & $\$$ & $3.715,00$ \\
\hline G.A.D. Municipal de Riobamba & $\$$ & - & $\$$ & $40.862 .593,81$ & $\$$ & - \\
\hline G.A.D. Municipal de Pallatanga & $\$$ & $16.849,95$ & $\$$ & $4.956 .548,63$ & $\$$ & $17.799,06$ \\
\hline G.A.D. Municipal de Latacunga & $\$$ & $750.000,00$ & $\$$ & $44.076 .515,57$ & $\$$ & $1.345 .170,87$ \\
\hline G.A.D. Municipal de Sigchos & $\$$ & $12.004,00$ & $\$$ & $8.589 .593,90$ & $\$$ & $12.004,00$ \\
\hline G.A.D. Municipal de Pasaje & $\$$ & $578.676,40$ & $\$$ & $16.438 .802,92$ & $\$$ & $478.282,34$ \\
\hline G.A.D. Municipal de Santa Rosa & $\$$ & $305.248,67$ & $\$$ & $20.669 .647,32$ & $\$$ & $190.951,30$ \\
\hline G.A.D. Municipal de Balsas & $\$$ & $32.150,64$ & $\$$ & $112.440,28$ & $\$$ & $1.879,97$ \\
\hline G.A.D. Municipal de Atacames & $\$$ & $287.121,00$ & $\$$ & $12.361 .696,23$ & $\$$ & $221.567,65$ \\
\hline G.A.D. Municipal de Yaguachi & $\$$ & $167.808,07$ & $\$$ & $12.602 .518,56$ & $\$$ & $167.808,07$ \\
\hline G.A.D. Municipal de Guay aquil & $\$$ & $17.913 .870,50$ & $\$$ & $791.525 .073,31$ & $\$$ & $17.913 .870,50$ \\
\hline G.A.D. Municipal de Palestina & $\$$ & $124.870,42$ & $\$$ & $3.742 .007,83$ & $\$$ & $108.733,67$ \\
\hline G.A.D. Municipal de Milagro & $\$$ & $987.116,65$ & $\$$ & $39.258 .612,21$ & $\$$ & $950.477,78$ \\
\hline G.A.D. Municipal deEl Triunfo & $\$$ & $567.716,00$ & $\$$ & $46.033 .604,08$ & $\$$ & $407.833,76$ \\
\hline G.A.D. Municipal de Durán & $\$$ & $3.648 .757,76$ & $\$$ & $63.006 .309,45$ & $\$$ & $3.213 .167,77$ \\
\hline G.A.D. Municipal de San Miguel de Ibarra & $\$$ & $2.573 .507,45$ & $\$$ & $43.317 .100,09$ & $\$$ & $1.905 .464,96$ \\
\hline G.A.D. Municipal de Cotacachi & $\$$ & $180.000,00$ & $\$$ & $12.961 .008,41$ & $\$$ & $140.600,00$ \\
\hline G.A.D. Municipal de Antonio Ante & $\$$ & $273.971,35$ & $\$$ & $16.601 .168,10$ & $\$$ & $268.654,46$ \\
\hline G.A.D. Municipal de Gonzanamá & $\$$ & $20.000,00$ & $\$$ & $4.976 .822,71$ & $\$$ & $11.670,21$ \\
\hline G.A.D. Municipal de Sozoranga & $\$$ & $4.000,00$ & $\$$ & $4.176 .244,54$ & $\$$ & $1.792,55$ \\
\hline G.A.D. Municipal de Baba & $\$$ & $14.809,73$ & $\$$ & $9.377 .813,78$ & $\$$ & $14.809,73$ \\
\hline G.A.D. Municipal deChone & $\$$ & $257.790,64$ & $\$$ & $19.931 .808,71$ & $\$$ & $231.267,63$ \\
\hline G.A.D. Municipal de San Vicente & $\$$ & $37.943,12$ & $\$$ & $6.046 .772,00$ & $\$$ & $37.943,12$ \\
\hline G.A.D. Municipal de Portoviejo & $\$$ & $2.860 .000,00$ & $\$$ & $66.193 .583,27$ & $\$$ & $1.846 .408,11$ \\
\hline G.A.D. Municipal de Thaisha & $\$$ & $5.000,00$ & $\$$ & $9.271 .530,21$ & $\$$ & $3.673,12$ \\
\hline G.A.D. Municipal de Pablo Sexto & $\$$ & $2.530,65$ & $\$$ & $2.882 .844,25$ & $\$$ & $2.500,93$ \\
\hline G.A.D. Municipal de Archidona & $\$$ & $69.953,64$ & $\$$ & $14.359 .556,45$ & $\$$ & $56.743,59$ \\
\hline G.A.D. Municipal de Carlos Julio Arosemena Tola & $\$$ & $35.750,00$ & $\$$ & $4.028 .553,16$ & $\$$ & $33.013,08$ \\
\hline G.A.D. Municipal de Fran cisco de Orellana & $\$$ & $1.608 .804,42$ & $\$$ & $54.489 .501,41$ & $\$$ & $1.236 .756,83$ \\
\hline Distrito metropolitano deQuito & $\$$ & $42.520 .000,00$ & $\$$ & $626.929 .571,10$ & $\$$ & $41.025 .371,18$ \\
\hline G.A.D. Municipal Rumiñahui & $\$$ & $1.900 .000,00$ & $\$$ & $34.041 .775,58$ & $\$$ & $1.947 .049,78$ \\
\hline G.A.D. Municipal de Santo Domingo & $\$$ & $1.919 .715,62$ & $\$$ & $167.958 .443,79$ & $\$$ & $1.701 .623,55$ \\
\hline G.A.D. Municipal de Shushufindi & $\$$ & $897.805,00$ & $\$$ & $25.195 .550,25$ & $\$$ & $652.503,98$ \\
\hline G.A.D. Municipal de Cevallos & $\$$ & $37.983,38$ & $\$$ & $4.917 .873,64$ & $\$$ & $32.001,29$ \\
\hline G.A.D. Municipal de Palanda & $\$$ & $17.773,26$ & $\$$ & $4.935 .030,31$ & $\$$ & $15.053,35$ \\
\hline G.A.D. Municipal de Yacuambi & $\$$ & $2.635,21$ & $\$$ & $4.201 .721,95$ & $\$$ & $1.463,49$ \\
\hline G.A.D. Municipal de Puebloviejo & $\$$ & $49.561,00$ & $\$$ & $8.381 .920,73$ & \$ & $49.561,00$ \\
\hline G.A.D. Municipal de Mera & $\$$ & $29.468,95$ & $\$$ & $8.267 .816,31$ & $\$$ & $29.468,95$ \\
\hline
\end{tabular}

Fuente: Cédula presupuestaria de ingresos extraída de páginas web de cada Municipio. 
Dentro del estudio se planteó el criterio hipotético, de manera que la hipótesis alternativa enunció que, la recaudación de patentes incide en el presupuesto de entidades gubernamentales. Mientras que, la hipótesis nula mencionó que, la recaudación de patentes no incide en el presupuesto de entidades gubernamentales. Para contrastar el supuesto, se aplicó pruebas estadísticas paramétricas conocidas como: coeficiente de correlación de Pearson que permite definir la variable con mayor influencia y la aplicación metodológica de la regresión lineal mediante el programa R-Studio que es un entorno de desarrollo integrado que permite manejar $\mathrm{R}$ (Software diseñado para realizar análisis estadísticos y gráficas) que logra sintetizar el proceso estadístico para el conocimiento de los resultados [15].

\section{IV.RESULTADOS}

\section{A..Patentes y Presupuesto Municipal}

Las variables de investigación en el presente artículo son ingresos totales del municipio, recaudación y asignación presupuestaria (presupuestado) del impuesto a las patentes del año 2017. Al aplicar el coeficiente de correlación de Pearson, se midió la fuerza de correlación lineal entre los valores x e y de la muestra (Tabla I), para determinar, si existe o no, una relación lineal [16]. Por lo tanto, se obtuvo una correlación de 0,99 lo que representa una relación lineal directa entre lo presupuestado y lo recaudado (Ver tabla II).

\section{TABLA II. Matriz de correlación de Pearson}

\begin{tabular}{lrrr}
\hline & \multicolumn{1}{c}{ Ingresos Totales } & \multicolumn{1}{c}{ Recaudado } & Presupues tado \\
\hline Ingres os Totales & 1 & & \\
Recaudado & 0,867152601 & 1 & \\
Pres upuestado & 0,860783452 & 0,99942265 & 1 \\
\hline
\end{tabular}

Al conocer las variables con mayor relación, se procedió a aplicar un modelo de regresión lineal mediante el software estadístico R-Studio, que refuerza la correlación obtenida con una pendiente positiva de 1,03 como coeficiente en la variable independiente (bX); es decir, existe una relación directa positiva; en otras palabras, al momento que la recaudación aumente mayor será el valor del presupuesto.

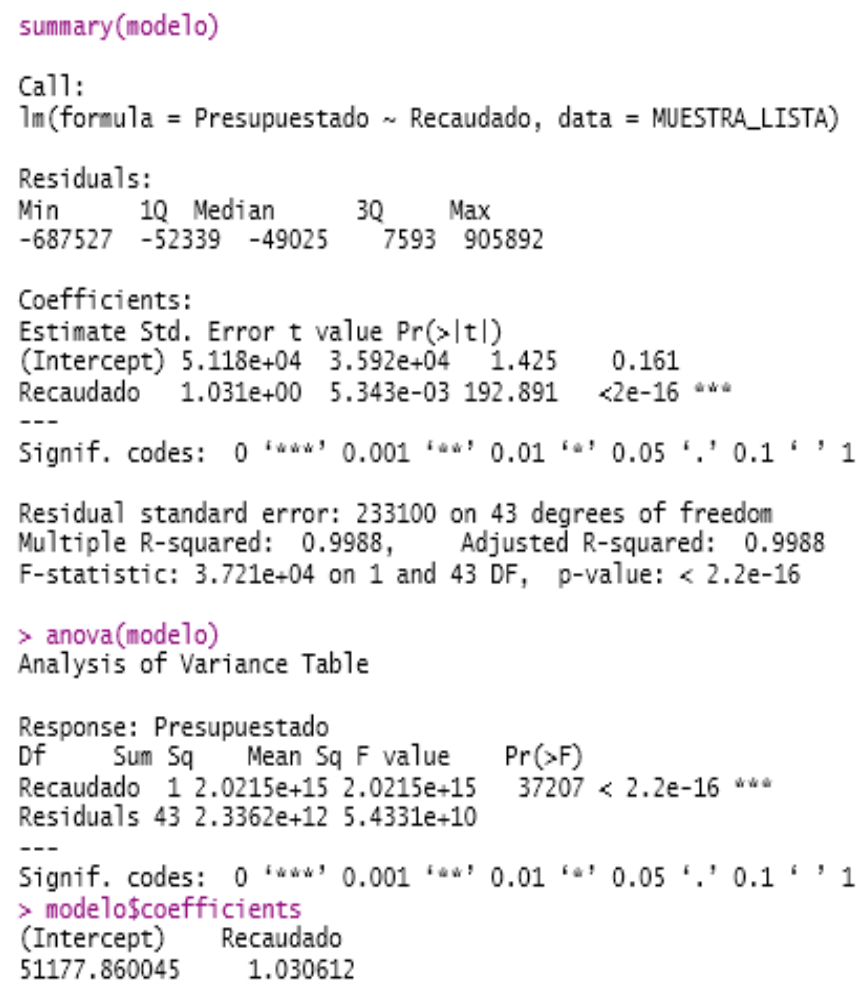

Fig. 4. Regresión lineal 
Estadísticamente, los valores muestran una relación positiva y al identificar el coeficiente de determinación R2, se obtiene una bondad de ajuste del $99,88 \%$, es decir, los valores que están cerca del modelo estadístico son mayores. Por tanto, la probabilidad de que la variable dependiente tienda a cambiar por una variación del coeficiente de regresión es alta. Se obtuvo un P-valor menor de $0,05(0,00)$, se procede a rechazar la hipótesis nula y aceptar la alterna, es decir, que la recaudación de patentes si influye en el presupuesto de entidades gubernamentales. Por lo tanto, fue necesario conocer qué sec- tor económico es el que ha sido el mayor contributario con este tributo durante el periodo 2017.

\section{B.Análisis por sectores.}

Para el análisis de revisión por sectores, se consideró la información del Directorio de Empresas y Establecimientos (DIEE) (ver tabla III). El Servicio de Rentas Internas permitió conocer información detallada del número de empresas y establecimientos que existen en el Ecuador tomando como base las declaraciones tributarias realizadas en el año 2017 [14].

\section{TABLA III. Empresas por Sector Económico}

\begin{tabular}{|c|c|c|}
\hline \multicolumn{3}{|c|}{$\begin{array}{l}\text { Empresas según participación nacional (universo DIEE), año } 2017 \text { por sector } \\
\text { económico (Número y porcentaje) }\end{array}$} \\
\hline Sector Económico & $\begin{array}{c}\text { Nro. } \\
\text { Empresas }\end{array}$ & $\%$ Total \\
\hline TOTAL & 884.236 & $100,00 \%$ \\
\hline Servicios & 361.149 & $40,84 \%$ \\
\hline Comercio & 319.503 & $36,13 \%$ \\
\hline Agricultura, ganadería, silvicultura y pesca & 98.156 & $11,10 \%$ \\
\hline Industrias manufactureras & 73.474 & $8,31 \%$ \\
\hline Construcción & 29.829 & $3,37 \%$ \\
\hline Explotación de minas y canteras & 2.125 & $0,24 \%$ \\
\hline
\end{tabular}

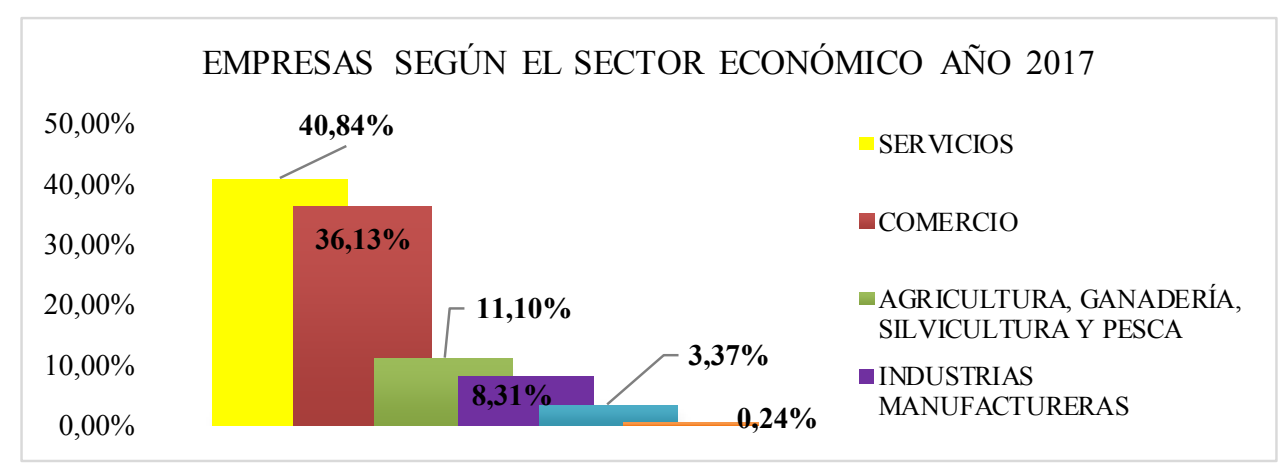

Fig. 5. Empresas por Sector Económico

Durante el año 2017 en el Ecuador, las entidades según su sector económico las de servicios tienen mayor participación cuantitativa en el mercado con un $40,84 \%$ y el comercial un $36,13 \%$, es decir, existen más empresas de servicios que comerciales, por tanto, para la aportación del impuesto de patentes estos dos grupos son los que más contribuyeron durante el periodo fiscal, dado que, es un requisito para su funcionamiento. Considerando el volumen de ventas, se obtiene la siguiente información (Ver tabla IV). 
TABLA IV. Ventas por Sector Económico

\begin{tabular}{lrrr}
\hline $\begin{array}{l}\text { Ventas totales según participación en el total (univers o DIEE), año 2017 por } \\
\text { s ector económico (dólares corrie ntes y porce ntaje) }\end{array}$ \\
\hline \multicolumn{1}{c}{ Sector Económico } & Ventas & Totales & \% Total \\
\hline TOTAL & $\$ \mathbf{1 5 8 . 5 6 7 . 1 8 8 . 7 5 3}$ & $\mathbf{1 0 0 , 0 0 \%}$ \\
Comercio & $\$$ & 60.430 .896 .176 & $38,11 \%$ \\
Servicios & $\$$ & 38.828 .319 .563 & $24,49 \%$ \\
Industrias manufactureras & $\$$ & 33.788 .580 .851 & $21,31 \%$ \\
Explotación de minas y canteras & $\$$ & 9.952 .310 .026 & $6,28 \%$ \\
Agricultura, ganadería, silvicultura y pesca & $\$$ & 8.862 .008 .862 & $5,59 \%$ \\
Construcción & $\$$ & 6.705 .073 .275 & $4,23 \%$ \\
\hline
\end{tabular}

VENTAS TOTALES POR SECTOR ECONÓMICO AÑO 2017

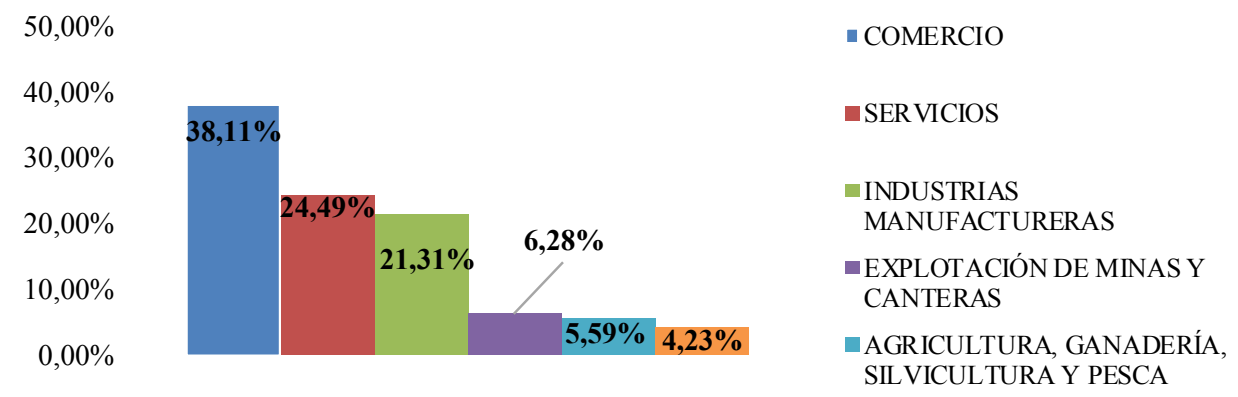

Fig. 6. Ventas totales por Sector Económico

Las ventas de las entidades durante el año 2017 en el Ecuador según su sector económico muestran que la mayor participación tiene el sector comercial con un $38,11 \%$, las de servicios con el $24,49 \%$ y las industrias manufactureras con el 21,31\%, lo quiere decir que, para fines tributarios sobre la renta de las personas naturales o jurídicas, mayor aportación tiene el sector comercial.

\section{V.CONCLUSIONES}

Utilizando herramientas estadísticas como el coeficiente de correlación de Pearson y R-Studio se pudo determinar que las variables de investigación están estrechamente relacionadas, por tanto, la influencia existe, siempre y cuando el proceso de recaudación sea eficiente. En este proceso existe una serie de problemas debido a que en la sociedad la gran mayoría de personas no cuenta con cultura tributaria, afectando directamente al ingreso que percibe las diferentes entidades gubernamentales provocando que estos no sean autónomos, sino que dependan del estado para cumplir con sus funciones, por esta razón, los Gobiernos Autónomos Descentralizados toman en cuenta este tributo al momento de desarrollar su presupuesto debido a que es un ingreso corriente percibido por todos los negocios dentro de su jurisdicción territorial.

Las patentes como obligaciones tributarias influyen en el presupuesto de las entidades gubernamentales, tal es el caso de los Gobiernos Autónomos Descentralizados como responsables del cobro de este tributo; al analizar las empresas por sectores económicos en el país según número de establecimientos, primero se encuentran ubicados los del sector de servicios y al notar el volumen de ventas en el año de los cuales se consideran los impuestos, los que mayores aportaciones tendrían son los comerciales; por ende, se concluye que el sector comercial y de servicio realizaron mayores contribuciones tributarias durante el año 2017, teniendo en cuenta que el impuesto a las patentes es un tributo de carácter obligatorio para el funcionamiento de estos sectores.

\section{REFERENCIAS}

[1]G. Galarza, S. Andrade, y T. Cabezas, «Ley de Propiedad Intelectual. Registro Oficial No 320,» Correos del Ecuador. Quito, Ecuador. LPI. 1998. [En línea]. Available: https:/www.correosdelecuador.gob.ec/ wp-content/uploads/downloads/2015/05/LEY_DE_ 
PROPIEDAD_INTELECTUAL.pdf.

[2]F. Cordero y F. Vergara, "Código Orgánico de Organización Territorial Descentralizado. Registro Oficial Suplemento 303 de 19-oct.-2010,» Ministerio de Gobierno. Quito, Ecuador. (COOTAD). 2018. [En línea]. Available: http://www.ambiente.gob.ec/wp-content/ uploads/downloads/2018/05/COOTAD.pdf.

[3]Ministerio de Economía y Finanzas, «Reglamento del Código Orgánico de Planificación y Finanzas Públicas. Registro Oficial Suplemento 383 de 26-nov.-2014,» 2018. [En línea]. Available: https://www.igualdadgenero.gob.ec/wp-content/uploads/2019/02/Reglamento-del-Código-Orgánico-de-Planificaicón-y-Finanzas. pdf.

[4]M. Vera, y J. Martínez, «Propuesta para incrementar la recaudación del impuesto anual por patente para el Gobierno Autónomo Descentralizado Ilustre Municipalidad del Cantón Daule.,» 2018. [En línea]. Available: http://repositorio.ulvr.edu.ec/bitstream/44000/2079/1/T-ULVR-1880.pdf.

[5]K. Talacera, «Estrategias Jurídicas y Tributarias para incrementar la recaudación del Impuesto a la Patente en el GAD Municipal de Tulcán.,» Tesis de maestría. Universidad Central del Ecuador. Quito, Ecuador. 2019.

[En línea]. Available: http://www.dspace.uce.edu.ec/ bitstream/25000/20209/1/T-UCE-0003-CAD-075-P. pdf.

[6]L. Villacis, "Gestión de la recaudación del impuesto a la patente municipal y su impacto en el presupuesto financiero en el GAD Municipalidad de Ambato período 2016.,» tesis de Maestría. Universidad Técnica de Ambato. Ambato, Ecuador. 2018. [En línea]. Available: https://repositorio.uta.edu.ec/bitstream/123456789/27587/3/T4234M.pdf.

[7]T. Sanclemente, «Recaudación del impuesto de patente a los contribuyentes del Gobierno Autónomo Descentralizado Municipal del Cantón Eloy Alfaro (GADMEA),» tesis de Maestría. Pontificia Universidad Catolica del Ecuador Sede Ambato. Ambato, Ecuador. 2018. [En línea]. Available: https://repositorio.pucesa. edu.ec/bitstream/123456789/1517/1/SANCLEMENTE CAMPAZ TANYA DAYANARA.pdf.

[8]G. Urgilés y K. Chávez, «Evolución de la recaudación tributaria y gasto público en el ecuador durante la ultima década,» Visión Empresarial, nº 7, pp. 139-155, 2017.

[9]E. Cando, y C. Cárdenas «La correcta fijación del impuesto a la patente municipal y el derecho del contribuyente frente a la falta de homologación de leyes por la discrepancia de la ordenanza 022-2010 frente a la Constitución y el COOTAD, en el cantón Rumiñahui durante el año 201,» 2016. [En línea]. Available: http://
www.dspace.uce.edu.ec/bitstream/25000/6908/3/TUCE-0013-Ab-301.pdf.

[10]Asamblea Nacional Constituyente, «Constitución de la República del Ecuador. Registro ofcial 449 de 20 Oct. 2008,» 2018. [En línea]. Available: http://www.ambiente.gob.ec/wp-content/uploads/downloads/2018/09/ Constitucion-de-la-Republica-del-Ecuador.pdf.

[11]R. Paredes y B. Pinda, «Análisis de la Política Tributaria en la Recaudación Fiscal: caso Ecuador,» Episteme, vol. 5, $\mathrm{n}^{\mathrm{o}}$ Especial, pp. 674-688, 2018.

[12] S. Domínguez, y N. Guayllas, «Determinación de la base imponible para el impuesto de patentes municipales a las actividades económicas cuyos propietarios son personas naturales no obligadas a llevar contabilidad en el cantón Cuenca.,» tesis de Maestría. Universidad Católica de Cuenca. Cuenca, Ecuador 2014.

[13]J. Guarco, «El proceso de recaudación de las patentes municipales y su impacto en el presupuesto del Gobierno Autónomo Descentralizado del cantón Riobamba durante el período 2014.,» .,» Tesis de Maestría. UNACH, Chimborazo, Ecuador. 2016. [En línea]. Available: http://dspace.unach.edu.ec/bitstream/51000/1493/1/UNACH-FCP-CPA-2016-0011. pdf.

[14]Instituto Nacional de Estadística y Censos, «Directorio de Empresas y Establecimientos 2017,» 2018. [En línea]. Available: https://www.ecuadorencifras.gob.ec/ documentos/web-inec/Estadisticas_Economicas/DirectorioEmpresas/Directorio_Empresas_2017/Documentos_DIEE_2017/Documentos_DIEE_2017/Principales_Resultados_DIEE_2017.pdf.

[15]L. Ballesteros, «Posibilidades interactivas de R como entorno de trabajo para un análisis dinámico de datos,» 2017. [En línea]. Available: http://tauja.ujaen. es/jspui/bitstream/10953.1/6767/1/Trabajo_Fin_de_ Grado_.pdf.

[16]M. Triola, Estadística. México. 12va Edición: Pearson Educación, 2013.

[17]J. Paz, y M. Cepeda, «Historia de los Impuestos en Ecuador. Visión sobre el régimen impositivo en la historia económica nacional.,» 2015. Servicio de Rentas Internas. Quito, Ecuador. [En línea]. Available: http:// www.sri.gob.ec/web/guest/home.

[18]J.Torres, «Reforma al Código Municipal en el que se encuentra la ordenanza que regula el impuesto de patentes municipales en el cantón Santo Domingo a fin de incorporar una sección que contemple el ejercicio de la facultad determinadora.,» Tesis de Maestría. UNIANDES, Santo Domingo, Ecuador. 2017. [En línea]. Available: http://dspace.uniandes.edu.ec/bitstream/123456789/7877/1/PIUSDAB024-2018.pdf. 


\section{RESUMEN CURRICULAR}

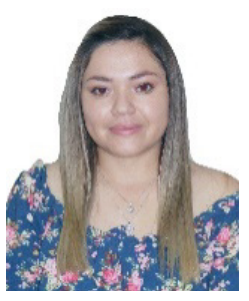

Salazar Nicole, sus estudios universitarios lo realizó en la Universidad de Las Fuerzas Armadas Sede Latacunga del departamento de Ciencias Económicas Administrativas y del Comercio. Es candidata a Ingeniera en Finanzas y Auditoria.

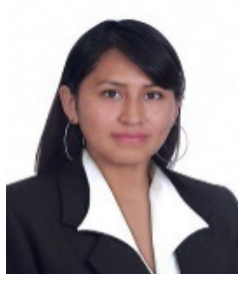

Vega Jessica, sus estudios universitarios lo realizó en la Universidad de Las Fuerzas Armadas Sede Latacunga del departamento de Ciencias Económicas Administrativas y del Comercio. Es candidata a Ingeniera en Finanzas y Auditoria.

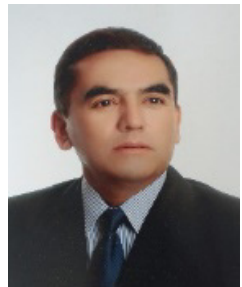

Parreño Carlos, Master en Dirección de Empresas, Economista, Abogado. Docente de la Universidad de las Fuerzas Armadas ESPE Sede Latacunga. 